\title{
A Discriminator with a Current-Sum Multiplicity Output for the PHENIX Multiplicity Vertex Detector
}

R. S. Smith, E. J. Kennedy, R. G. Jackson

University of Tennessee, Knoxville

M. L. Simpson, C. L. Britton, W. L. Bryan, U. Jagadish, G. R. Young

Oak Ridge National Laboratory

B.V. Jacak, J. Kapustinsky

Los Alamos National Laboratory

A. Oscarson

University of Lund, Sweden

:

309030

$08 \mathrm{TI}$

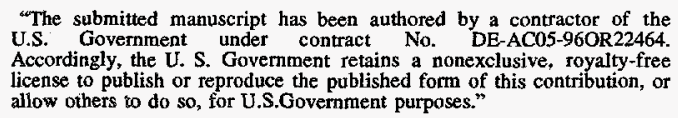

, the U. Government retains a nonexclusive, royalty-free allow others to do so, for U.S.Government purposes."

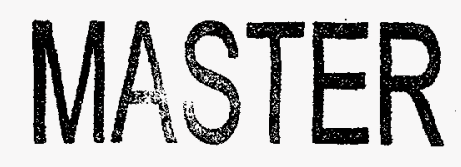

DESTRIBUTON OF THS DOCUMENT IS UNMMTEO कh

* Research sponsored by the U.S. Department of Energy and performed at Oak Ridge National Laboratory, managed by Lockheed Martin Energy Research Corporation for the U.S. Department of Energy under Contract No. DE-AC05-96OR22464. 


\section{DISCLAIMER}

This report was prepared as an account of work sponsored by an agency of the United States Government. Neither the United States Government nor any agency thereof, nor any of their employees, makes any warranty, express or implied, or assumes any legal liability or responsibility for the accuracy, completeness, or usefulness of any information, apparatus, product, or process disclosed, or represents that its use would not infringe privately owned rights. Reference herein to any specific commercial product, process, or service by trade name, trademark, manufacturer, or otherwise does not necessarily constitute or imply its endorsement, recommendation, or favoring by the United States Government or any agency thereof. The views and opinions of authors expressed herein do not necessarily state or reflect those of the United States Government or any agency thereof. 


\section{DISCLAMMER}

Portions of this document may be illegible in electronic image products. Images are produced from the best available original document. 


\title{
A Discriminator with a Current-Sum Multiplicity Output for the PHENIX Multiplicity Vertex Detector ${ }^{1}$
}

\author{
R. S. Smith, E. J. Kennedy, R. G. Jackson \\ University of Tennessee, Knoxville \\ M. L. Simpson, C. L. Britton, W. L. Bryan, U. Jagadish, G. R. Young \\ Oak Ridge National Laboratory \\ B. V. Jacak, J. Kapustinsky \\ Los Alamos National Laboratory \\ A. Oscarson \\ University of Lund, Sweden
}

\begin{abstract}
A current output multiplicity discriminator for use in the front-end electronics (FEE) of the Multiplicity Vertex Detector (MVD) for the PHENIX detector at RHIC has been fabricated in the a $1.2-\mu$ CMOS, $n$-well process. The discriminator is capable of triggering on input signals ranging from $0.25 \mathrm{MIP}$ to 5 MIP. Frequency response of the discriminator is such that the circuit is capable of generating an output for every bunch crossing (105 ns) of the RHIC collider. Channel-to-channel threshold matching was adjustable to $\pm 4 \mathrm{mV}$. One channel of multiplicity discriminator occupied an area of $85 \mu \times 630 \mu$ and consumed $515 \mu \mathrm{W}$ from a single 5-V supply. Details of the design and results from prototype device testing are presented.
\end{abstract}

\section{INTRODUCTION}

The Multiplicity Vertex Detector (MVD) is the inner most detector of PHENIX. The MVD consists of $\sim 34,000$ channels of charge sensitive preamplifiers, analog memory units (AMU), 11-bit ADCs and multiplicity discriminators which are used primarily to determine collision vertex and event multiplicity. By summing the outputs of many multiplicity discriminators, a value proportional to the number of colliding particles is created. This information aids in the generation of a Level-1 trigger $[1,2]$. Figure 1 depicts the system diagram for one channel of MVD front-end electronics (FEE).

The high channel count coupled with the small physical area in which the FEE reside require a low-power, small-area, multiplicity discriminator implementation. A design goal of approximately $500 \mu \mathrm{W} /$ channel was established as an upper limit for power consumption. Additionally, due to detector

\footnotetext{
${ }^{1}$ Research sponsored by the U.S. Department of Energy and performed at the Oak Ridge National Laboratory, managed by Lockheed Martin Energy Research Corporation for the U.S. Department of Energy under contract DE-AC05-960R22464.
}

pitch constraints, the discriminator (as well as all other MVD FEE) must be arrayable within an $85 \mu$ pitch.

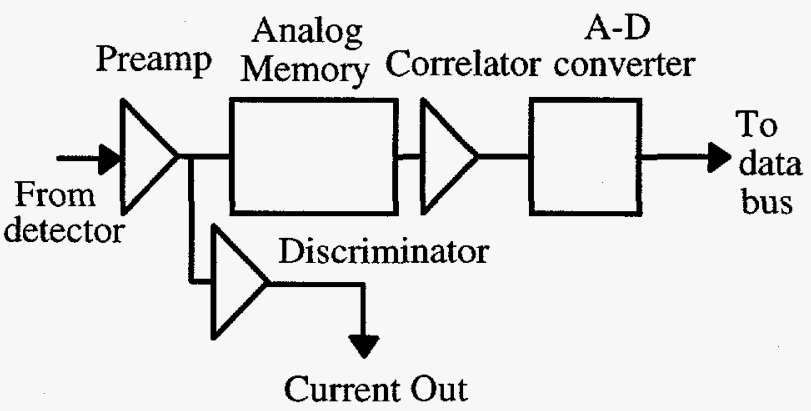

Figure 1: Block diagram of MVD FEE.

Another aspect of the high channel count is the need to have a common discriminator threshold setting for 32-channels of multiplicity discriminator located on a single die. This goal requires that the discriminator be extremely well matched over all process variations. For this reason, methods of offset elimination have been examined.

Timing constraints for the multiplicity discriminator are dictated by the RHIC bunch crossing interval, which has been established as $105 \mathrm{~ns}$ in order to achieve the highest possible luminosity. Within every $105 \mathrm{~ns}$ cycle, the discriminator is capable of providing valid event information.

\section{Multiplicity Discriminator Design}

Since the discriminator receives input signals directly from a charge sensitive preamplifier, it must operate with step inputs. Each applied step consists of a fixed risetime pulse (60 ns) with varying amplitudes. Removing the long decay tail of the preamplifier signal requires differentiation. Both passive and active differentiation topologies were considered. Passive differentiation is easy to implement and well matched across channels. However, for the discriminator to be active during every bunch crossing, the differentiation time constant must be kept small which leads to severe signal attenuation. Such high levels of signal attenuation place further constraints on the 


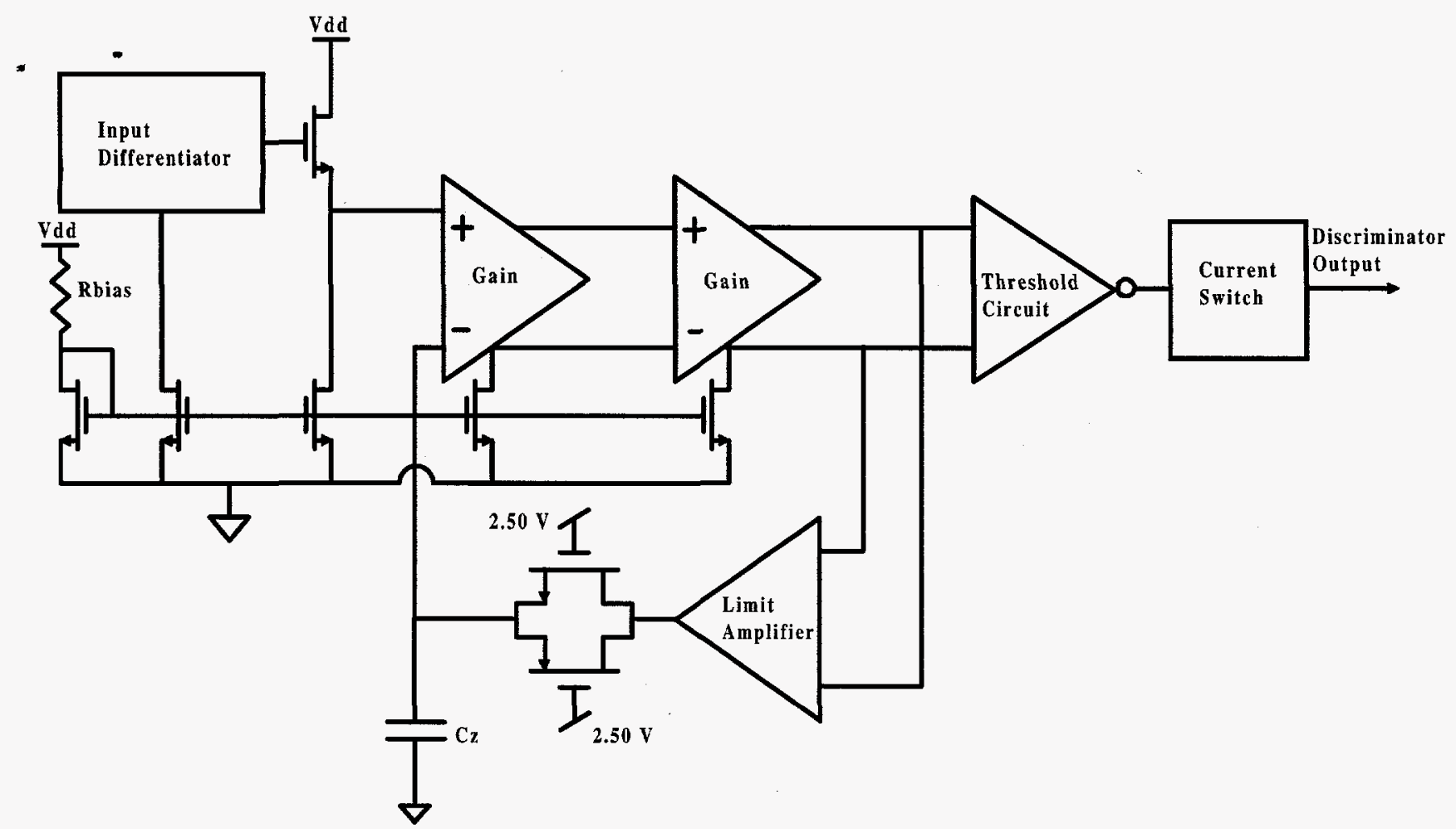

Figure 3: Complete diagram for 1 channel of multiplicity discriminator.

remaining circuitry. However, active differentiation provides an adequate frequency response as well as signal amplification.

Typical active differentiators utilizing operational amplifiers are inappropriate for this application because of size constraints. An acceptable compromise to the area, power, speed and matching specifications was selected and is shown in Figure 2. Devices M1 and M4 comprise a basic commongate stage whose gain is given by

$$
\mathrm{A}_{\mathrm{V}}=\mathrm{gm}_{\mathrm{cff}(\mathrm{M} 1)} \mathrm{R}_{\mathrm{DS}(\mathrm{M} 4)} \text {. }
$$

Signal differentiation is achieved through the C-R network generated by the $0.50 \mathrm{pF}$ capacitor and the relatively small resistance seen looking into the source of M1. M2 and M3 provide the necessary bias current for the differentiator. Through adjustment of the voltage applied to the gate of the M4 device biased in the ohmic region, the amount of signal gain may be varied.

The circuit of Figure 2 is especially susceptible to channelto-channel mismatch resulting from random process variations. The major sources of error stem from the channel-to-channel variations produced by drain current mismatch in M2 and transconductance mismatch in M1. It has been shown that transconductance matching is directly proportional to the WL product [4]. Thus, maximizing the area of M1 minimizes the gain error associated with gm mismatch. Transistors M2a and M2b provide a means by which the differentiator bias current can be varied from approximately $10 \mu \mathrm{A}$ to $20 \mu \mathrm{A}$ by turning n-channel switches, M2sa and M2sb, either on (logic high) or off (logic low). By adjusting the differentiator bias current for each channel, channel-to-channel mismatch can be reduced.

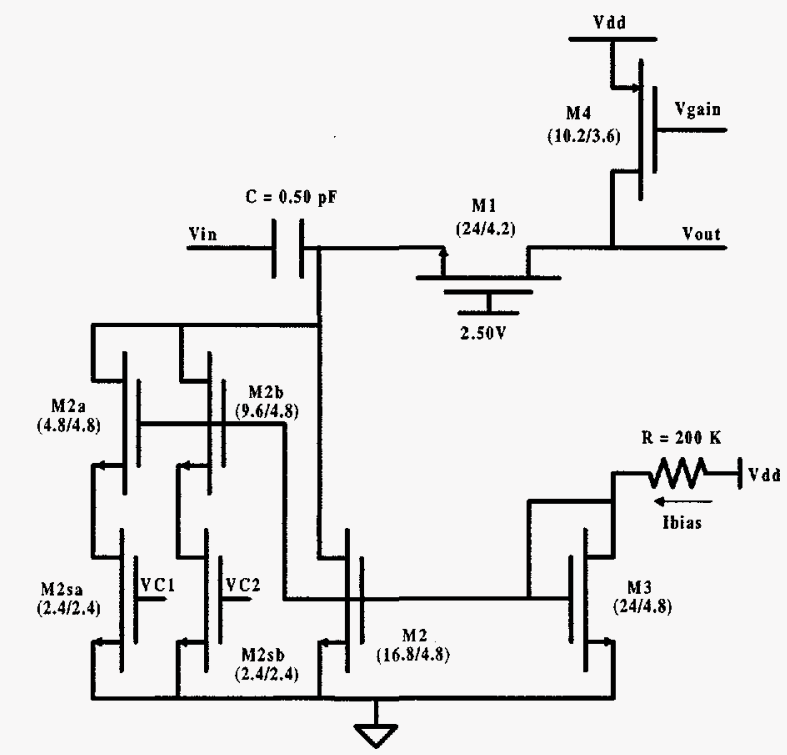

Figure 2: Input differentiator topology.

The remainder of the multiplicity discriminator, illustrated in the block diagram of Figure 3, consists of two gain stages, a dc feedback circuit to eliminate offsets, a threshold circuit, and an output current switch. Each gain stage is composed of a differential single-stage amplifier with a gain of $5 \mathrm{~V} / \mathrm{V}$.

After amplification, the resulting differential signal is applied to the threshold circuit of Figure 4 . The threshold detect circuit of Figure 4 is similar to one described previously and channel-to-channel matching on the order of $\pm 3 \mathrm{mV}$ has been observed $[3,5]$. 


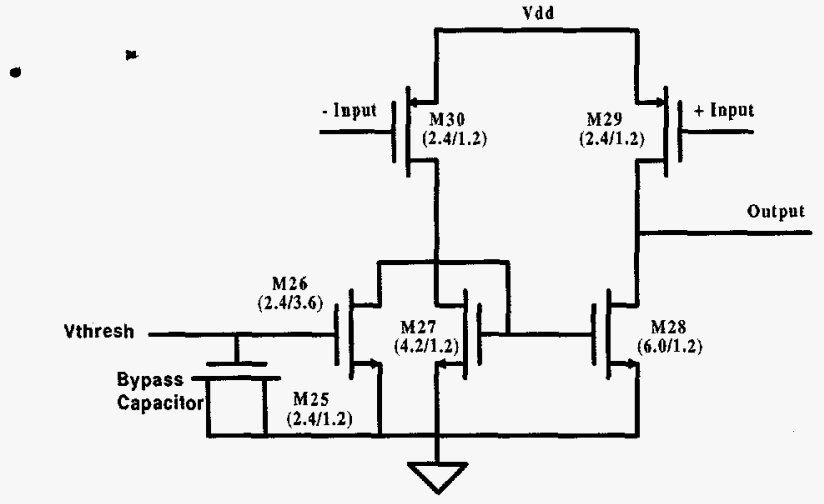

Figure 4: Threshold adjustment circuit.

Mismatch between the input devices of the gain stages introduces an offset voltage. Achieving the highest possible channel-to-channel matching requires dc feedback to eliminate this offset. Continuous dc feedback was selected to eliminate offsets because of the low event rate expected for the MVD.

To maintain circuit stability required implementation of an $8 \mathrm{pF}$ hold capacitor within the dc feedback loop. A high density capacitor structure, whose cross-section is shown in Figure 5, facilitated inclusion of this large device while still maintaining the stringent area requirements of the discriminator. The capacitor combines the densities obtained by poly-poly and MOS capacitor structures. This combined density can approach $2 \mathrm{fF} / \mu^{2}$ while maintaining a series resistance of less than $30 \Omega$.

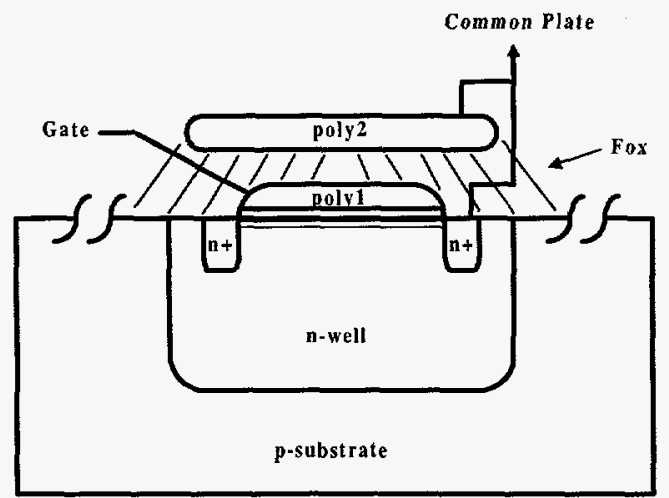

Figure 5: High density capacitor structure used to implement the 8 $\mathrm{pF}$ hold capacitor.

The final element required to implement the function of multiplicity discrimination is the current switch output circuit. To convert the output voltage into an acceptable current suitable for summing, the topology of Figure 6 was utilized. Transistors M1 - M4 comprise the bias and reference voltage common to 32-channels of discriminator. When the discriminator triggers (logic low), M6 turns on and allows a nominal current of $30 \mu \mathrm{A}$ to flow through M5 and onto the current summing bus.

\section{RESULTS}

An 8-channel prototype version of the multiplicity discriminator proposed for use within the MVD was evaluated based on the system requirements previously discussed. One

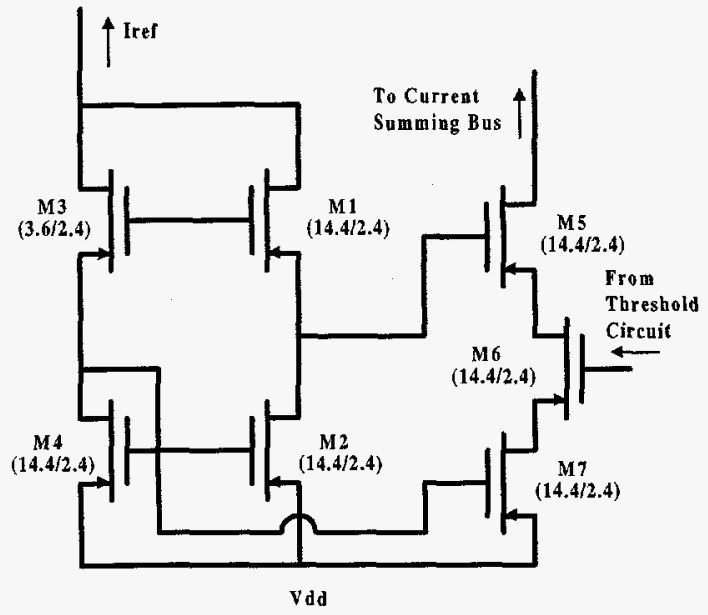

Figure 6: Output current switch topology.

channel occupied an area of $85 \mu \times 630 \mu$ and consumed roughly $515 \mu \mathrm{W}$ from a single 5-V supply.

The multiplicity discriminator was tested with a $60 \mathrm{~ns}$ risetime signal supplied from a LeCroy 9210 pulse generator. As shown in Figure 7 , the discriminator was capable of triggering on $20-\mathrm{mV}$ input signals while providing an appropriate timing response. Additionally, through proper manipulation of the input differentiator gain adjustment and the threshold adjustment, the discriminator could be adjusted such that inputs greater than $450 \mathrm{mV}$ were required to trigger the circuit. The graph of Figure 8 illustrates the triggering characteristics of the discriminator for various settings of these externally adjustable bias voltages.

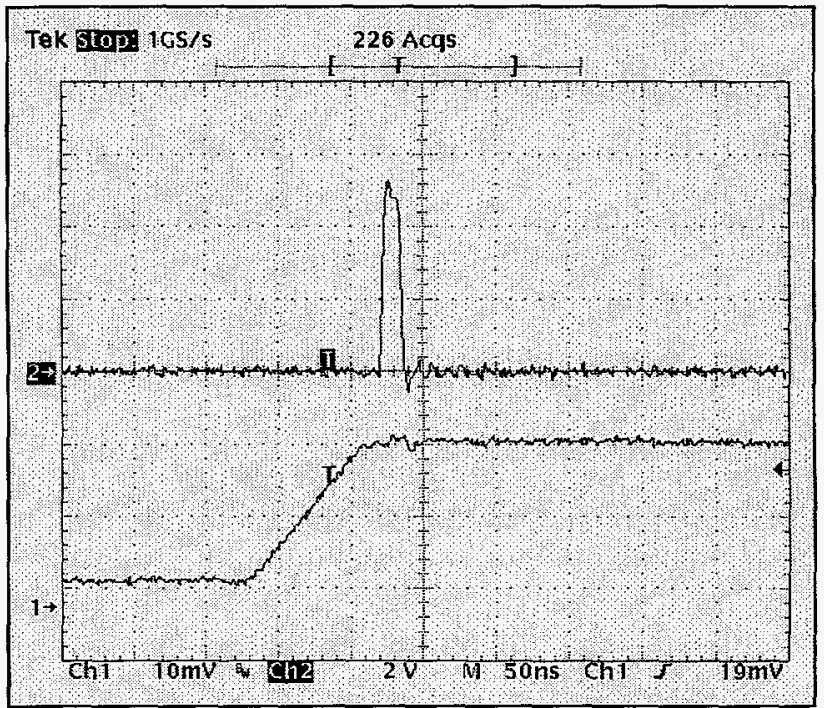

Figure 7: Sample discriminator output.

Two methods were used to evaluated the channel-tochannel matching characteristics. The first evaluation of the discriminator threshold matching characteristics was performed with a fixed input differentiator bias current of 18 $\mu \mathrm{A}$. The average threshold voltage mismatch for the 5 prototype chips tested was $\pm 8.1 \mathrm{mV}$. The alternative evaluation utilized the individual channel current adjustment illustrated in Figure 2. Implementation of this adjustment 
resulted in a reduction of channel-to-channel mismatch to \pm $42 \mathrm{mV}$. Table 1 provides a comparison of the threshold mismatch with and without adjustment for the 5 sample chips.

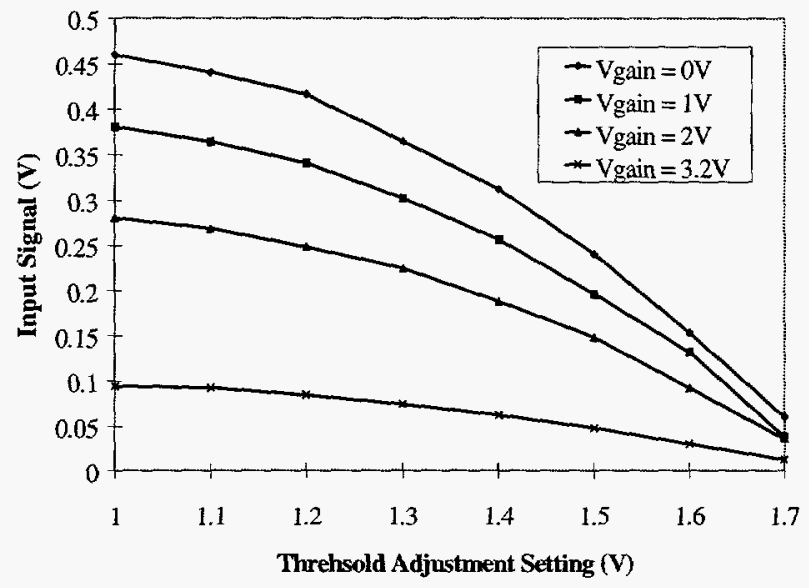

Figure 8: Input required to trigger discriminator as a function of gain and threshold setting.

Table 1.

Threshold mismatch of prototype chips with and without adjustment.

\begin{tabular}{|c|c|c|}
\hline Chip & $\begin{array}{c}\text { Threshold } \\
\text { Matching without } \\
\text { Adjustment }\end{array}$ & $\begin{array}{c}\text { Threshold } \\
\text { Matching with } \\
\text { Adjustment }\end{array}$ \\
\hline 1 & $\pm 7.75 \mathrm{mV}$ & $\pm 3 \mathrm{mV}$ \\
\hline 2 & $\pm 5.25 \mathrm{mV}$ & $\pm 4 \mathrm{mV}$ \\
\hline 3 & $\pm 9.25 \mathrm{mV}$ & $\pm 5 \mathrm{mV}$ \\
\hline 4 & $\pm 7 \mathrm{mV}$ & $\pm 4.5 \mathrm{mV}$ \\
\hline 5 & $\pm 11.25 \mathrm{mV}$ & $\pm 4.5 \mathrm{mV}$ \\
\hline Average & $\pm 8.1 \mathrm{mV}$ & $\pm 4.2 \mathrm{mV}$ \\
\hline
\end{tabular}

\section{CONCLUSIONS}

A small area, arrayable multiplicity discriminator suitable for use in the MVD at RHIC has been presented. This circuit provides a highly flexible design which allows for compensation of random processing mismatch over multiple channels. The circuit establishes a low-power, small-area means of direct integration with a charge sensitive preamplifier.

\section{ACKNOWLEDGMENTS}

The authors gratefully acknowledge T. Subich and L. Anthony of the Oak Ridge National Laboratory for their contributions to the completion of this paper.

\section{REFERENCES}

[1] Kehoe, W. L., et al., PHENIX Conceptual Design Report. 29 Jan. 1993.

[2] Britton, C. L., et al., "Low Noise, Low Power Dissipation LSI Electronics for Heavy Ion Detectors". Conference Record of 1996 IEEE International Symposium on Circuits and Systems, Vol. 1, pp. 133-136.
[3] Simpson, M. L., et al., "A Monolithic, Constant-Fraction Discriminator Using Distributed R-C Delay Line Shaping," IEEE Transactions on Nuclear Science, Vol. 43, No. 3, June 1996, pp. 1695-1699.

[4] Lakshmikumar, K. R., et al., "Characterization and Modeling of Mismatch in MOS Transistors for Precision Analog Design," IEEE Journal of Solid-State Circuits, Vol. SC-21, No. 6, December 1986.

[5] Smith, R. S., "Development of a Multiplicity Discriminator for High Energy Physics Experiments," Masters Thesis, University of Tennessee, Knoxville, December 1996. 